Biotempo (Lima)

ORIGINAL ARTICLE / ARTÍCULO ORIGINAL

ACUTE TOXICITY OF THREE PESTICIDES (BUTACHLOR, COPPER OXYCHLORIDE AND CHLORPYRIFOS) ON THE MARINE BENTHIC AMPHIPOD APOHYALE GRANDICORNIS (KROYER, 1845) (CRUSTACEA: HYALIDAE)

\title{
TOXICIDAD AGUDA DE TRES PLAGUICIDAS (BUTACLOR, OXICLORURO DE COBRE Y CLORPIRIFOS) SOBRE EL ANFÍPODO BENTÓNICO MARINO APOHYALE GRANDICORNIS (KROYER, 1845) (CRUSTACEA: HYALIDAE)
}

\author{
Diana Lina Sotelo-Vásquez ${ }^{1} \&$ José Iannacone $^{1,2}$
}

\footnotetext{
Laboratorio de Parasitología. Facultad de Ciencias Biológicas. Universidad Ricardo Palma (URP). Lima, Perú. E-mail: joseiannacone@gmail.com

2 Laboratorio de Ecología y Biodiversidad Animal (LEBA). Universidad Nacional Federico Villarreal (UNFV). E-mail: joseiannacone@gmail.com

Author for correspondence: joseiannacone@gmail.com
}

\begin{abstract}
Pesticides have been widely used since ancient times in agriculture for the protection of crops from plagues. However, most of these generate high environmental pollution. Despite of law regulation, they continue to arrive in different ways to the ocean. The present work evaluates the toxicity of three commercial pesticides in Peru: the herbicide Butachlor, the insecticide Chlorpyrifos and the fungicide Copper Oxychloride, on the marine amphipod Apohyale grandicornis (Krøyer, 1845). Ecotoxicological bioassays were carried out in each one with standardized protocols, in addition to Potassium Dichromate sensitivity test. Likewise, based on acute test, the environmental risk of the three pesticides at the aquatic level was evaluated by calculating the risk quotient (RQs). According to the $\mathrm{LC}_{50}$ of the pesticides the sequence in descended order of toxicity was: Butachlor> Chlorpyrifos> Copper Oxychloride. For the Potassium Dichromate sensitivity test, an $\mathrm{LC}_{50}$ value of $13.04 \mathrm{mg} \cdot \mathrm{L}^{-1}$ was found. The herbicide Butachlor registered the highest toxicity of all the pesticides evaluated at the $96 \mathrm{~h}$ of exposure in $A$. grandicornis with $\mathrm{LC}_{50}$ of $0.019 \mathrm{mg} \cdot \mathrm{L}^{-1}$, showing a toxicity 1.11 times higher than Chlorpyrifos and 28.42 more than Copper Oxychloride. Finally, both Butachlor and Chlorpyrifos present an environmental risk on $A$. grandicornis. While, Copper Oxychloride was an environmental risk when using the safety factor to calculate the risk quotient.
\end{abstract}

Keywords: amphipod - Apohyale grandicornis - Butachlor - Chlorpyrifos - Copper Oxychloride - environmental risk - Potassium Dichromate 


\section{RESUMEN}

Los plaguicidas han sido enormemente utilizados desde tiempos antiguos, en la agricultura para la protección de cultivos del ataque de plagas. Sin embargo, la mayoría de estos genera una alta contaminación ambiental; y a pesar de la regulación, estos siguen llegando por diferentes vías a las aguas marinas. El presente trabajo evaluó la toxicidad de tres plaguicidas de uso comercial en el Perú: el herbicida Butaclor, el insecticida Clorpirifos y el fungicida Oxicloruro de Cobre, sobre el anfípodo marino Apohyale grandicornis (Krøyer, 1845). Se realizaron bioensayos ecotoxicológicos con los tres modelos empleando protocolos estandarizados, además se efectuó una prueba de sensibilidad utilizando Dicromato de Potasio. Asimismo, tomando como base los ensayos agudos, se evaluó el riesgo ambiental de los tres plaguicidas a nivel acuático, mediante el cálculo del cociente de riesgo (CR). La secuencia de mayor a menor toxicidad según la $\mathrm{CL}_{50}$ (Concentración Letal media) de los plaguicidas fue: Butaclor $>$ Clorpirifos> Oxicloruro de Cobre. Para la prueba de sensibilidad con Dicromato de Potasio se halló un valor de $\mathrm{CL}_{50}$ de 13,04 mg. $\mathrm{L}^{-1}$. El herbicida Butaclor registró la mayor toxicidad de todos los plaguicidas evaluados a las $96 \mathrm{~h}$ de exposición en $A$. grandicornis con $\mathrm{CL}_{50}$ de $0,019 \mathrm{mg} \cdot \mathrm{L}^{-1}$ mostrando una toxicidad 1,11 veces mayor que el Clorpirifos y 28,42 más que el Oxicloruro de Cobre. Finalmente tanto el Butaclor como el Clorpirifos presentan un riesgo ambiental sobre A. grandicornis; mientras que el Oxicloruro de cobre sólo mostró riesgo ambiental al utilizar el factor de seguridad para el cálculo del Cociente de riesgo.

Palabras clave: anfípodo - Apohyale grandicornis - Butaclor - Clorpirifos - Dicromato de Potasio - Oxicloruro de Cobre - riesgo ambiental

\section{INTRODUCCIÓN}

El aumento en las necesidades de producción de alimentos y de otros productos de la agricultura, debido al crecimiento poblacional, entre otros factores, ha llevado a la producción y aplicación masiva de productos químicos para el control de las plagas, originando así problemas de tipo ambiental (Cyrino et al., 2004; Ferreira et al., 2004; Albert, 2005).

Tres tipos de plaguicidas: un herbicida (Butaclor), un insecticida (Clorpirifos) y un fungicida (Oxicloruro de cobre), están dentro de los principales plaguicidas formulados e importados en el Perú. Guerrero (2007) comenta que el Butaclor (N-butoxymetil-2-cloro2',6'-dietilacetanilida) es un herbicida residual, de acción pre-emergente que controla malezas anuales, monocotiledóneas como ciperáceas, commelináceas y gramíneas; además de algunas hoja ancha, en el cultivo de arroz. El Clorpirifos $\left(\mathrm{C}_{9} \mathrm{H}_{11} \mathrm{Cl}_{3} \mathrm{NO}_{3} \mathrm{PS}\right)$ es considerado un insecticida organofosforado de amplio espectro. Originalmente tenía un uso residencial, para el control de plagas en césped y plantas ornamentales. Sin embargo a partir de la década de los setenta este uso se amplifico al sector agrícola; siendo utilizado en granos, frutas, nueces y vegetales (Leight \& Van Dolah, 1999). Como todos los fungicidas, el Oxicloruro de cobre $\left(\mathrm{Cl}_{2} \mathrm{Cu}_{4} \mathrm{H}_{6} \mathrm{O}_{6}\right)$ es un compuesto químico que se encarga de eliminar o evitar el desarrollo de hongos (Pérez \& Forbes, 2007).
Los plaguicidas, transportados por los ríos, llegan finalmente a las zonas marinas que poseen una gran importancia, debido a que son fuente de alimento y hábitat para la fijación y desarrollo de una gran diversidad de organismos bentónicos. Los plaguicidas y otras sustancias químicas pueden ocasionar efectos ecotoxicológicos en la biota acuática (Leight \& Van Dolah, 1999; Iannacone \& Alvariño, 2000; Abessa \& Souza, 2003; Cyrino et al., 2004; Ferreira et al., 2004; Tilak et al., 2007; Lasheidani et al., 2008; Abigail et al., 2015).

Los anfípodos peracáridos en los ambientes marinos, presentan alta abundancia y diversidad (Serejo, 2003; Chapman, 2007; Pérez, 2009; Cadien, 2015; Navarro, 2015). También, juegan un rol ecológico relevante en los ambientes intermareales, y están involucrados en la conversión de energía facilitando su flujo entre los productores primarios (algas, plantas epífitas), detritos y consumidores superiores. Apohyale grandicornis (Krøyer, 1845) (Hyalidae) puede considerarse una especie de anfípodo adecuada para la evaluación de riesgos ambientales marinos (King et al., 2006).

La evaluación de los efectos de los plaguicidas ayudará a conocer como los organismos acuáticos podrían verse afectados por estas sustancias químicas (Palma et al., 2008; Yin et al., 2008; He et al., 2012; Nwani et al., 2013). Los resultados obtenidos permiten establecer criterios de calidad y legislación para la protección de los ambientes acuáticos donde residen estos organismos (Iannacone \& 
Alvarińo, 2009; Liu et al. 2011; Giddings et al., 2014; Gradila, 2015; Paredes \& Anaya, 2015; Lewis et al., 2016).

Por lo señalado, el objetivo de esta investigación fue determinar la toxicidad aguda de tres plaguicidas comerciales a base de Butaclor, Clorpirifos y Oxicloruro de Cobre, en el anfípodo bentónico marino $A$. grandicornis.

\section{MATERIALES Y MÉTODOS}

Área de Estudio

Ejemplares de $A$. grandicornis fueron colectados en la playa “La arenilla" ( $12^{\circ} 04^{\prime} 26,34$ ”'S, $77^{\circ} 09^{\prime} 44,18^{\prime}$ O), en el distrito de la Punta, Provincia constitucional del Callao, Perú, cuando el nivel de mareas oscilaba entre una altitud de 10-20 cm. La playa se caracterizó por presentar un ambiente rocoso de grava mediana y con presencia de algas.

\section{Apohyale grandicornis}

La especie fue identificada por Analí Jiménez Campeán en el Laboratorio de bentos marino, del Instituto de Mar del Perú (IMARPE), quién se basó en las claves taxonómicas de Bousfield \& Hendrycks (2002) y Chapman (2007). Se complementó la identificación de la especie en el Laboratorio de Parasitología (Lab-79) de la Facultad de Ciencias Biológicas, Universidad Ricardo Palma (URP), utilizando la clave taxonómica propuesta por Gonzales (1991).

La colecta de los ejemplares se realizó mediante tamices de 300 y $500 \mathrm{u}$, donde quedaron retenidos los anfípodos. Posteriormente los organismos fueron colocados en baldes de plástico de $8 \mathrm{~L}$ de capacidad, con agua de mar, para su traslado al laboratorio húmedo de Ecotoxicología acuática del IMARPE; en donde fueron separados y aclimatados.

\section{Plaguicidas empleados}

Se utilizaron tres tipos de plaguicidas, representativos del mercado Nacional: el fungicida a base de Oxicloruro de cobre (Cupravit OB21); el herbicida a base de Butachlor (Arroba 600EC) y el insecticida a base de Clorpirifos (Tifón 2,5 PS). Estos plaguicidas fueron adquiridos en la tienda "Inversiones Agrícola M \& V SAC", localizada en el distrito de San Luis, Lima, Perú.

\section{Aclimatación}

Se llevó a cabo en el laboratorio de Ecotoxicología del IMARPE, donde se procedió a separar manualmente los anfípodos en 3 piscinas de $10 \mathrm{~L}$ de la siguiente manera: hembras y machos (adultos), hembras y machos (juveniles) y hembras grávidas (Peluso, 2011). Se mantuvieron a los anfípodos con agua del lugar de colecta, que gradualmente se fue remplazando por agua de mar filtrada obtenida en el IMARPE. Se dio un tiempo de aclimatación de 7 días, siendo los organismos alimentados con la microalga Nannochloropsis oculata D.J. Hibberd, 1981 hasta un día antes del bioensayo. Esta microalga fue proporcionada por el Laboratorio de cultivo de microalgas del IMARPE. Los organismos se mantuvieron a una temperatura de $20^{\circ} \mathrm{C} \pm 2$ y con oxigenación constante no menor a $6 \mathrm{mg} \cdot \mathrm{L}^{-1}$.

\section{Toxicidad aguda}

Se basaron en la metodología estandarizada propuesta por la USEPA (1994), y en otros trabajos realizados con anfípodos (Cesar et al., 2004; Fairchild et al., 2010; Peluso, 2011). La prueba de toxicidad aguda para $A$. grandicornis se evaluó en 5 concentraciones con cuatro réplicas, incluyendo el control, en un Diseño en Bloques Completamente Randomizado (DBCR). Los bioensayos tuvieron una duración de exposición de 96 $h$, en condiciones semiestáticas. Manteniéndose a una temperatura $>19^{\circ} \mathrm{C}$, mediante una mesa de agua de temperatura controlada; así como una oxigenación suave (> $\left.6 \mathrm{mg} \cdot \mathrm{L}^{-1}\right)$, mediante un sistema de aireación.

Para las diluciones se utilizó agua de mar filtrada, proporcionada por el IMARPE. Los organismos no fueron alimentados durante el periodo de exposición a los plaguicidas. Se utilizaron envases de vidrio de 200 $\mathrm{mL}$, conteniendo $54 \mathrm{~g}$ de sedimento (grava mediana a grande, debidamente esterilizada con Hipoclorito de Sodio al $10 \%$ ) y $150 \mathrm{~mL}$ de los plaguicidas diluidos. En cada envase se colocaron 10 anfípodos con tallas entre 3 a $5 \mathrm{~mm}$. Estos microcrustáceos fueron expuestos a los tres plaguicidas en las siguientes concentraciones: Oxicloruro de cobre: 500, 50, 5 y $0,5 \mathrm{mg} \cdot \mathrm{L}^{-1}$ (Güven et al., 1999; Cyrino et al., 2004; Ferreira et al., 2004; Fryday \& Thompson, 2012); Butaclor: 4, 0,4, 0,004 y 0,0004 mg.L-1 (Tilak et al., 2007; Liu et al., 2011; He et al., 2012; Nwani et al., 2013), y Clorpirifos: 1; 0,1; 0,001 y $0,0001 \mathrm{mg} \cdot \mathrm{L}^{-1}$ (Leight \& Van Dolah, 1999; Iannacone \& Alvarińo, 2002, 2004; Iannacone et al., 2002; CCME, 2008; Montagna, 2010).

Además de las 4 concentraciones, todos los bioensayos contaron con un control, a base de agua de mar filtrada, con cuatro repeticiones. El indicador de la prueba de toxicidad aguda fue la mortandad, los cuales al presentar inmovilidad fueron extraídos, con la ayuda de una pipeta de plástico, y observados en el estereoscopio por un lapso de 1 min aproximadamente. Sólo aquellos individuos que 
no realizaron ninguna movilidad fueron considerados muertos. Los bioensayos se consideraron válidos cuando la mortandad no superó al 10\% (USEPA, 1994;
Iannacone \& Alvarińo, 2004). En la siguiente tabla 1 se presenta un resumen de las condiciones de los bioensayos de toxicidad.

Tabla 1. Resumen de condiciones del ensayo con Apohyale grandicornis.

\author{
Agitación \\ Tiempo de exposición \\ Aireación \\ Agua de dilución \\ Número de organismos por unidad de prueba \\ Número de réplicas por concentraciones \\ Número de organismos por concentración \\ Alimentación \\ Concentraciones de prueba \\ Respuesta \\ Criterio de aceptabilidad de la prueba
}

\section{Sensibilidad con Dicromato de Potasio}

Para la elaboración del control positivo se utilizó, el tóxico de referencia, Dicromato de potasio $\left(\mathrm{K}_{2} \mathrm{Cr}_{2} \mathrm{O}_{7}\right)$ (USEPA, 2002). Se realizaron cinco diluciones a concentraciones de 1000, 100, 10, 1, 0,1 mg. $\mathrm{L}^{-1}$ (Rudolph et al., 2011). Además de un control, el cual constó de agua de mar filtrada. Cada dilución, incluido el control, constó de cuatro réplicas.

\section{Parámetros fisicoquímicos}

Se tomaron los siguientes parámetros básicos: $\mathrm{pH}$, salinidad, oxígeno disuelto (OD) y temperatura. El $\mathrm{pH}$, la temperatura y el OD se monitorearon mediante un multiparámetro $\mathrm{HANNA}^{\oplus}$ con dos sensores (OD/ $\mathrm{T}^{\circ}$ y $\left.\mathrm{pH} / \mathrm{T}^{\circ}\right)$. La salinidad por otro lado se midió con un refractómetro HAND HELD ${ }^{\circledR}$. Tanto el pH como la salinidad se midieron al inicio (instalación) y al término del bioensayo. Mientras que la temperatura y el OD se midieron diariamente.

\section{Evaluación del riesgo ambiental (ERA)}

Se empleó esta técnica para determinar la naturaleza y magnitud de riesgo de los plaguicidas evaluados, empleando los escenarios más críticos y de mayor exposición, utilizando al anfípodo $A$. grandicornis para determinar el riesgo en organismos acuáticos.

\section{Concentración ambiental estimada (CAE)}

Para estimar el valor de CAE se utilizó la tabla elaborada

No
$96 \mathrm{~h}$
Constante
Agua de mar estéril
10 organismos
4 réplicas
40 individuos
No requiere alimentación
5 y 1 control negativo
Muerte (inmovilización total por $15 \mathrm{~s}$ )
$>90 \%$ sobrevivientes en los controles

por la CA (2002), para lo cual se estimó la dosis máxima recomendada ( $\mathrm{kg}$ i.a.ha ${ }^{-1}$ ) para cada uno de los tres productos agrícolas utilizados. Del mismo modo se tomó la profundidad recomendada de $2 \mathrm{~m}$.

\section{Estimación de la concentración prevista sin efecto (PNEC)}

Se calculó el valor de PNEC utilizando la fórmula propuesta por Planes \& Fuchs (2015), la cual es siguiente: $\mathrm{CL}_{50} / \mathrm{FS}=\mathrm{PNEC}$

Las $\mathrm{CL}_{50}$ s fueron tomados de los tres plaguicidas a las $96 \mathrm{~h}$ de exposición para $A$. grandicornis. El factor de seguridad (FS) fue de 1000.

\section{Cálculo de los Coeficientes de riesgo (CR)}

Se calculó dos CR, uno utilizando la CAE y otro mediante los valores de PNEC y el FS. Para hallar el CR mediante el CAE y el $\mathrm{CL}_{50}$ se siguió la recomendación de diferentes autores (Iannacone \& Alvarińo, 2002; CA, 2002) quienes emplearon la siguiente formula:

Concentración ambiental estimada (CAE) /

Concentración letal media $\left(\mathrm{CL}_{50}\right)=\mathrm{CR}$

Mientras que para el cálculo del CR por medio del PNEC se usó la fórmula utilizada por Planes \& Fuchs, (2015), la cual es la siguiente: Concentración ambiental estimada $(\mathrm{CAE}) / \mathrm{PNEC}=\mathrm{CR}$ 


\section{Procesamiento de datos}

Para el análisis de la $\mathrm{CL}_{50}$ se recurrió a la $2^{\text {da }} \mathrm{Ed}$ de la hoja de cálculo Excel Probit basado en Finney (1952), En aquellos casos donde los datos arrojados fueron anómalos se procedió a usar el programa de análisis de relación de toxicidad (TRAP). Para la validación de los ensayos de toxicidad individual se utilizó el paquete estadístico SPSS versión 24,0 para Windows 8,0. De este modo, se calculó las diferencias de las mortalidades evaluadas mediante el estadístico ANOVA y a un nivel de significancia de $\mathrm{p} \leq 0,05$. Asimismo, se calcularon los valores de LOEC (Concentración más baja con efecto observado) y NOEC (Concentración sin efecto observado) para cada plaguicida usado, a las 24, 48, 72 y 96 h en base a la prueba de Turkey.

\section{Lugar de Ejecución}

La parte experimental de la tesis se llevó a cabo en el Laboratorio de Ecotoxicología acuática del IMARPE, Chucuito-Callao, Perú (Lat. 12 $04^{\prime}$ 1,197” S Long. 77º 09' 28.331" W) a una elevación de $6 \mathrm{msnm}$. Mientras que el trabajo de gabinete se realizó en el Laboratorio de Parasitología (Lab-79) de la Universidad Ricardo Palma
(URP), Santiago de Surco, Lima, Perú. (Lat. 12 ${ }^{\circ} 7^{\prime}$ 59,16" S; Long. $76^{\circ} 58^{\prime}$ 49,8" W).

\section{Aspectos éticos}

Los autores declaran que se cumplió con toda la normatividad ética nacional e internacional.

\section{RESULTADOS}

\section{Parámetros fisicoquímicos}

En la Tabla 2, se muestran los promedios registrados de las condiciones fisicoquímicas básicas a las que fueron sometidos los anfípodos en los diferentes bioensayos. Se observó para el caso de la temperatura rangos entre 17,94 $\pm 1,94$ y $21,82 \pm 2,64^{\circ} \mathrm{C}$ que correspondieron al Oxicloruro y al Clorpirifos, respectivamente. De igual forma se observa que el oxígeno disuelto varió poco presentando rangos entre 6,78 \pm 0,44 y 7,01 \pm 0,08 mg. $\mathrm{L}^{-1}$ Oxicloruro de Cobre y el Dicromato de Potasio. El pH también mostró poca variación con rangos de 8,05 \pm 0,05 y 8,46 \pm 0,08 del Butaclor y Clorpirifos. Por último la salinidad si mostró variación entre los ensayos con un rango de 29,58 \pm 0,65 y 40,06 \pm 3,92 \%o para el Oxicloruro de Cobre y el Butaclor.

Tabla 2. Promedio de parámetros fisicoquímicos básicos para los bioensayos ecotoxicológicos con tres plaguicidas y dicromato de potasio en $A$. grandicornis.

\begin{tabular}{cccccc}
\hline \multicolumn{2}{l}{ Parámetros fisicoquímicos } & Butaclor & Clorpirifos & Oxicloruro de Cobre & Dicromato de Potasio \\
\hline Temperatura & ${ }^{\circ} \mathrm{C}$ & $20,21 \pm 0.21$ & $21,82 \pm 2,64$ & $17,94 \pm 1,94$ & $21,06 \pm 1,74$ \\
Oxígeno Disuelto & $\mathrm{mg} \cdot \mathrm{L}^{-1}$ & $6,83 \pm 0.15$ & $6,83 \pm 0,25$ & $6,78 \pm 0,44$ & $7,01 \pm 0,08$ \\
pH & & $8,05 \pm 0.05$ & $8,46 \pm 0,08$ & $8,15 \pm 0,13$ & $8,09 \pm 0,64$ \\
Salinidad & $\%$ oo & $40,06 \pm 3.92$ & $34,33 \pm 2,98$ & $29,58 \pm 0,65$ & $31,52 \pm 0,74$ \\
\hline
\end{tabular}

\section{Bioensayos de toxicidad aguda}

Los tres plaguicidas evaluados en los bioensayos presentaron un efecto tóxico sobre el anfípodo $A$. grandicornis a las 24, 48, 72 y $96 \mathrm{~h}$. En ninguno de los casos la mortandad fue superior al $10 \%$.

\section{Butaclor}

En la tabla 3 se señalan los porcentajes de mortandad de A. grandicornis, en las cinco concentraciones del herbicida Butaclor a las diferentes horas (24-96 h), indicando las diferencias significativas en la mortalidad entre las concentraciones evaluadas, la concentración letal media $\left(\mathrm{CL}_{50}\right)$, así como los valores de NOEC y LOEC. 
Tabla 3. Toxicidad del herbicida Butaclor a las 24-96h sobre Apohyale grandicornis.

\begin{tabular}{ccccc}
\hline $\mathrm{mg} \cdot \mathrm{L}^{-1}$ & $24 \mathrm{~h}$ & $48 \mathrm{~h}$ & $72 \mathrm{~h}$ & $96 \mathrm{~h}$ \\
\hline 0 & $0 \mathrm{a}$ & $0 \mathrm{a}$ & $0 \mathrm{a}$ & $0 \mathrm{a}$ \\
0,0004 & $0 \mathrm{a}$ & $10 \mathrm{a}$ & $15 \mathrm{a}$ & $15 \mathrm{a}$ \\
0,004 & $13 \mathrm{a}$ & $15 \mathrm{ab}$ & $28 \mathrm{ab}$ & $33 \mathrm{ab}$ \\
0,04 & $28 \mathrm{a}$ & $43 \mathrm{~b}$ & $50 \mathrm{~b}$ & $58 \mathrm{~b}$ \\
0,4 & $100 \mathrm{~b}$ & $100 \mathrm{c}$ & $100 \mathrm{c}$ & $100 \mathrm{c}$ \\
4 & $100 \mathrm{~b}$ & $100 \mathrm{c}$ & $100 \mathrm{c}$ & $100 \mathrm{c}$ \\
$\mathrm{CL}_{50}$ & 0,47 & 0,13 & 0,04 & 0,019 \\
$\mathrm{CL}_{50}$ inf & 0,07 & 0,03 & 0,01 & 0,00 \\
$\mathrm{CL}_{50}$ sup & 2,58 & 0,64 & 0,24 & 0,09 \\
$\mathrm{NOEC}_{\mathrm{LOEC}}$ & 0,04 & 0,004 & 0,004 & 0,004 \\
$\mathrm{~F}$ & 0,4 & 0,04 & 0,04 & 0,04 \\
Sig. & 59,23 & 52,96 & 35,18 & 33,97 \\
\hline
\end{tabular}

Letras minúsculas iguales en una misma columna representan que no hay diferencia significativa entre los valores por la prueba de Turkey $(\mathrm{p} \leq 0,05)$. NOEC= Concentración sin efecto observado. $\mathrm{LOEC}=$ Concentración mínima con efecto observado. $\mathrm{CL}_{50}$ = Concentración letal media. Inf.= Límite inferior. Sup.= Límite superior. F= Prueba de Fisher. Sig.= Nivel de significancia.

De este modo, se apreció que el Butaclor tuvo efectos significativos $(\leq 0,05)$ sobre la mortandad a las $24,48,72$ y $96 \mathrm{~h}$, siendo la concentración de $0,4 \mathrm{mg} \cdot \mathrm{L}^{-1}$ donde se evidenció el efecto tóxico del Butaclor a las $24 \mathrm{~h}$ y de $0,04 \mathrm{mg} \cdot \mathrm{L}^{-1}$ en el resto de horas. Se observó el $100 \%$ de mortandad en todas las horas, a partir de la concentración de $0,4 \mathrm{mg} \cdot \mathrm{L}^{-1}$. Asimismo los menores valores de NOEC y LOEC fueron de 0,004 y $0,04 \mathrm{mg} \cdot \mathrm{L}^{-1}$ a las 48,72 y 96 $\mathrm{h}$, mientras que los valores más altos fueron de 0,04 y 0,4 $\mathrm{mg} \cdot \mathrm{L}^{-1}$ las $24 \mathrm{~h}$ de exposición.

En la misma tabla se aprecian los valores de $\mathrm{CL}_{50}$, y sus límites inferior y superior a las 24, 48, 72 y $96 \mathrm{~h}$, presentando el Butaclor rangos entre 0,47 y $0,02 \mathrm{mg} \cdot \mathrm{L}^{-1}$.

\section{Clorpirifos}

La tabla 4 muestra los porcentajes de mortandad de $A$. grandicornis, de las concentraciones del insecticida Clorpirifos a las diferentes h (24-96 h), indicando las diferencias significativas en la mortalidad entre las concentraciones evaluadas, la concentración letal media $\left(\mathrm{CL}_{50}\right)$, así como los valores de NOEC y LOEC.

Tabla 4. Toxicidad del insecticida Clorpirifos a las 24-96h sobre Apohyale grandicornis.

\begin{tabular}{ccccc}
\hline $\mathrm{mg} \cdot \mathrm{L}^{-1}$ & $24 \mathrm{~h}$ & $48 \mathrm{~h}$ & $72 \mathrm{~h}$ & $96 \mathrm{~h}$ \\
\hline 0 & $0 \mathrm{a}$ & $0 \mathrm{a}$ & $0 \mathrm{a}$ & $2,5 \mathrm{a}$ \\
0,0001 & $0 \mathrm{a}$ & $0 \mathrm{a}$ & $0 \mathrm{a}$ & $2,5 \mathrm{a}$ \\
0,001 & $0 \mathrm{a}$ & $5 \mathrm{a}$ & $8 \mathrm{a}$ & $10 \mathrm{a}$ \\
0,01 & $0 \mathrm{a}$ & $8 \mathrm{a}$ & $8 \mathrm{a}$ & $13 \mathrm{a}$ \\
0,1 & $50 \mathrm{~b}$ & $93 \mathrm{~b}$ & $98 \mathrm{~b}$ & $98 \mathrm{~b}$ \\
1 & $78 \mathrm{c}$ & $98 \mathrm{~b}$ & $98 \mathrm{~b}$ & $100 \mathrm{~b}$ \\
$\mathrm{CL}_{50}$ & 0,10 & 0,02 & $\mathrm{ND}$ & 0,02 \\
$\mathrm{CL}_{50}$ inf & 0,03 & 0,01 & $\mathrm{ND}$ & 0,01 \\
$\mathrm{CL}_{50}$ sup & 0,37 & 0,05 & $\mathrm{ND}$ & 0,05 \\
$\mathrm{NOEC}_{\mathrm{LOEC}}$ & 0,01 & 0,01 & 0,01 & 0,01 \\
$\mathrm{~F}$ & 0,1 & 0,1 & 0,1 & 0,1 \\
Sig. & 65,47 & 224,63 & 339,12 & 233,23 \\
\hline
\end{tabular}


Letras minúsculas iguales en una misma columna representan que no hay diferencia significativa entre los valores por la prueba de Turkey $(\mathrm{p} \leq 0,05)$. NOEC $=$ Concentración sin efecto observado. $\mathrm{LOEC}=$ Concentración mínima con efecto observado. $\mathrm{CL}_{50}=$ Concentración letal media. Inf.= Límite inferior. Sup.= Límite superior. F= Prueba de Fisher. Sig.= Nivel de significancia. $\mathrm{ND}=$ No determinado.

De tal manera se observó que el Clorpirifos tuvo efectos significativos $(\leq 0,05)$ sobre la mortandad a las $24,48,72$ y $96 \mathrm{~h}$, siendo la concentración de $0,1 \mathrm{mg} \cdot \mathrm{L}^{-1}$ donde se evidenció el efecto tóxico del Clorpirifos en todas las horas analizadas. Se observó el $100 \%$ de individuos muertos a las $96 \mathrm{~h}$ a una concentración de $1 \mathrm{mg} \cdot \mathrm{L}^{-1}$. Asimismo los valores de NOEC y LOEC fueron de 0,01 y $0,1 \mathrm{mg} \cdot \mathrm{L}^{-1}$, para todas las h evaluadas.

En la misma tabla se aprecian los valores de $\mathrm{CL}_{50}$, y sus límites inferior y superior a las 24, 48 y $96 \mathrm{~h}$, presentando el Clorpirifos rangos entre 0,02 y $0,10 \mathrm{mg} \cdot \mathrm{L}^{-1}$.

\section{Oxicloruro de cobre}

En la tabla 5 se señalan los porcentajes de mortandad de A. grandicornis, de las seis concentraciones del fungicida Oxicloruro de Cobre a las diferentes horas (24-96 h), indicando las diferencias significativas en la mortalidad entre las concentraciones evaluadas, la concentración letal media $\left(\mathrm{CL}_{50}\right)$, así como los valores de NOEC y LOEC.

Tabla 5. Toxicidad del fungicida Oxicloruro de Cobre a las 24-94h sobre Apohyale grandicornis.

\begin{tabular}{|c|c|c|c|c|}
\hline \multirow{2}{*}{$\mathrm{mg} \cdot \mathrm{L}^{-1}$} & \multicolumn{4}{|c|}{$\%$} \\
\hline & $24 \mathrm{~h}$ & $48 \mathrm{~h}$ & $72 \mathrm{~h}$ & $96 \mathrm{~h}$ \\
\hline 0 & $0 \mathrm{a}$ & $0 \mathrm{a}$ & $2,5 a$ & $2,5 a$ \\
\hline 0,05 & $18^{a}$ & $25 \mathrm{ab}$ & $30 \mathrm{ab}$ & $42,5 \mathrm{ab}$ \\
\hline 0,5 & $13^{a}$ & $30 \mathrm{ab}$ & $43 \mathrm{ab}$ & $48 \mathrm{ab}$ \\
\hline 5 & $33^{a}$ & $43 \mathrm{ab}$ & $60 \mathrm{ab}$ & $63 \mathrm{~b}$ \\
\hline 50 & $35^{a}$ & $58 \mathrm{ab}$ & $65 \mathrm{ab}$ & $78 \mathrm{~b}$ \\
\hline 500 & $38^{\mathrm{a}}$ & $65 b$ & $75 b$ & $88 \mathrm{~b}$ \\
\hline $\mathrm{CL}_{50}$ & $>500^{*}$ & 17,87 & 3,30 & 0,54 \\
\hline $\mathrm{CL}_{50}{ }^{50} \mathrm{inf}$ & 107,22 & 0,98 & 0,20 & 0,05 \\
\hline $\mathrm{CL}_{50}$ sup & $>500$ & 326,59 & 54,13 & 6,46 \\
\hline NOEC & 500 & 50 & 50 & 0,5 \\
\hline LOEC & $>500$ & 500 & 500 & 5 \\
\hline F & 2,11 & 3,37 & 3,52 & 8,84 \\
\hline Sig. & 0,11 & 0,02 & 0,02 & 0,00 \\
\hline
\end{tabular}

Letras minúsculas iguales en una misma columna representan que no hay diferencia significativa entre los valores por la prueba de Turkey $(\mathrm{p} \leq 0,05)$. NOEC= Concentración sin efecto observado. $\mathrm{LOEC}=$ Concentración mínima con efecto observado. $\mathrm{CL}_{50}=$ Concentración letal media. Inf.= Límite inferior. Sup.= Límite superior. $\mathrm{F}=$ Prueba de Fisher. Sig.= Nivel de significancia. ${ }^{*}$ Se obtuvo un valor demasiado alto porque no se obtuvo más del $50 \%$ de mortandad.

Así, se apreció que el Oxicloruro de Cobre tuvo efectos significativos $(\leq 0,05)$ sobre la mortandad a las 48,72 y $96 \mathrm{~h}$, siendo la concentración de $0,05 \mathrm{mg} \cdot \mathrm{L}^{-1}$ donde se evidenció el efecto tóxico de este a las 48, 72 y 96 h. La mayor mortandad (88\%) se obtuvo $96 \mathrm{~h}$ a una concentración de $500 \mathrm{mg} \cdot \mathrm{L}^{-1}$, no llegando al $100 \%$ de individuos muertos en ningún tratamiento. Asimismo los valores de NOEC y LOEC fueron de 50 y $500 \mathrm{mg} \cdot \mathrm{L}^{-1}$ para las 48 y $72 \mathrm{~h}$, mientras que a las $96 \mathrm{~h}$ fueron de $0,5 \mathrm{y} 5 \mathrm{mg} \cdot \mathrm{L}^{-1}$. No se obtuvieron diferencias significativas (Sig.= 0,11 ) a las $24 \mathrm{~h}$. En esta tabla también se aprecian los valores de $\mathrm{CL}_{50}$, y sus límites inferior y superior a las $24,48,72$ y 96 h, presentando el Oxicloruro de Cobre rangos entre 0,54 a $>500 \mathrm{mg} \cdot \mathrm{L}^{-1}$.

\section{Prueba con Dicromato de Potasio}

Los resultados de la evaluación de la sensibilidad con Dicromato de Potasio en los ensayos de A. grandicornis se muestran en la tabla 6 . 
Tabla 6. Toxicidad del Dicromato de Potasio a las 24-96h sobre Apohyale grandicornis.

\begin{tabular}{ccccc}
\hline $\mathrm{mg} \cdot \mathrm{L}^{-1}$ & $24 \mathrm{~h}^{\mathrm{a}}$ & $48 \mathrm{~h}^{\mathrm{a}}$ & $72 \mathrm{~h}$ & $96 \mathrm{~h}$ \\
\hline 0 & $0 \mathrm{a}$ & $0 \mathrm{a}$ & $0 \mathrm{a}$ & $2,5 \mathrm{a}$ \\
0,1 & $0 \mathrm{a}$ & $5 \mathrm{a}$ & $5 \mathrm{a}$ & $5 \mathrm{ab}$ \\
1 & $0 \mathrm{a}$ & $8 \mathrm{a}$ & $8 \mathrm{a}$ & $10 \mathrm{ab}$ \\
10 & $3 \mathrm{a}$ & $8 \mathrm{a}$ & $13 \mathrm{a}$ & $20 \mathrm{~b}$ \\
100 & $0 \mathrm{a}$ & $25 \mathrm{~b}$ & $78 \mathrm{~b}$ & $98 \mathrm{c}$ \\
1000 & $100 \mathrm{~b}$ & $100 \mathrm{c}$ & $100 \mathrm{c}$ & $100 \mathrm{c}$ \\
$\mathrm{CL}_{50}$ & 550,94 & 167,17 & 41,69 & 13,04 \\
$\mathrm{CL}_{50}$ inf & $\mathrm{ND}$ & $\mathrm{ND}$ & 11,37 & 4,71 \\
$\mathrm{CL}_{50}$ sup & $\mathrm{ND}$ & 334,75 & 152,82 & 36,08 \\
$\mathrm{NOEC}_{\mathrm{LOEC}}$ & 100 & 10 & 10 & 1 \\
$\mathrm{~F}$ & 1000 & 100 & 100 & 10 \\
Sig. & 1585,00 & 110,02 & 146,48 & 183,53 \\
\hline
\end{tabular}

Letras minúsculas iguales en una misma columna representan que no hay diferencia significativa entre los valores por la prueba de Turkey $(\mathrm{p} \leq 0,05)$. NOEC= Concentración sin efecto observado. LOEC= Concentración mínima con efecto observado. $\mathrm{CL}_{50}=$ Concentración letal media. Inf.= Límite inferior. Sup.= Límite superior. $\mathrm{F}=$ Prueba de Fisher. Sig.= Nivel de significancia. $\mathrm{ND}=$ no determinado. ${ }^{*}$ Se obtuvo un valor excesivamente alto debido a que no se obtuvo más del $50 \%$ de mortandad. ${ }^{a}=$ Se Utilizó el paquete estadístico TRAP.

Se encontraron efectos significativos $(\leq 0,05)$ sobre la mortandad a las $24,48,72$ y $96 \mathrm{~h}$, siendo la concentración de $1000 \mathrm{mg} \cdot \mathrm{L}^{-1}$ donde se evidenció el efecto tóxico de este a las $24 \mathrm{~h}$; de $100 \mathrm{mg} \cdot \mathrm{L}^{-1}$ a las 48 y $72 \mathrm{~h}$ y de $10 \mathrm{mg} \cdot \mathrm{L}^{-1}$ a las 96 h. Se observó el 100\% de individuos muertos a una concentración de $1000 \mathrm{mg} \cdot \mathrm{L}^{-1}$ en todas las horas analizadas. De igual forma, los mayores valores de NOEC y LOEC fueron de 100 y $1000 \mathrm{mg} \cdot \mathrm{L}^{-1}$ a las $24 \mathrm{~h}$, mientras que los valores más bajos se obtuvieron a las $96 \mathrm{~h}$ a concentraciones de 1 y $10 \mathrm{mg} \cdot \mathrm{L}^{-1}$. Asimismo, se aprecian los valores de $\mathrm{CL}_{50}$, y sus límites inferior y superior a las 48,72 y $96 \mathrm{~h}$, mostrando el Dicromato de Potasio rangos entre 13,04 y $550,94 \mathrm{mg} \cdot \mathrm{L}^{-1}$.

\section{Análisis Comparativo de toxicidad a las $96 \mathrm{~h}$}

De acuerdo con el análisis de las $\mathrm{CL}_{50}$ a las $96 \mathrm{~h}$, la toxicidad de los plaguicidas para $A$. grandicornis ordenados en forma descendente respecto a su toxicidad, fue: Butaclor $>$ Clorpirifos $>$ Oxicloruro de Cobre (tablas 3, 4 y 5). De esta manera, se encontró que a las 96 h, el Butaclor es 1,11 veces más tóxico que el Clorírifos y un 28,42 veces más que el Oxicloruro de Cobre. Mientras que el Clorpirifos presentó 25,71 veces más toxicidad que el Oxicloruro de Cobre. Utilizando la misma lógica (mayor-menor toxicidad) se ordenaron los valores de NOEC y LOEC, obteniendo para el LOEC la siguiente secuencia: Butaclor $\left(0,04 \mathrm{mg} \cdot \mathrm{L}^{-1}\right)>$ Clorpirifos $\left(0,1 \mathrm{mg} \cdot \mathrm{L}^{-}\right.$ $\left.{ }^{1}\right)>$ Oxicloruro de Cobre $\left(5 \mathrm{mg} \cdot \mathrm{L}^{-1}\right)$. Y para el NOEC: Butaclor $\left(0,004 \mathrm{mg} \cdot \mathrm{L}^{-1}\right)>$ Clorpirifos $\left(0,01 \mathrm{mg} \cdot \mathrm{L}^{-1}\right)>$ Oxicloruro de Cobre $\left(0,5 \mathrm{mg} \cdot \mathrm{L}^{-1}\right)$.

\section{Riesgo ambiental}

La tabla 7 y 8 muestran un resumen de la evaluación del riesgo ambiental (ERA) de los tres plaguicidas investigados sobre $A$. grandicornis. En la tabla 7, tomando como base los ensayos agudos, se encontró que tanto el Butaclor como el Clorpirifos presentaron un elevado riesgo ambiental sobre A. grandicornis. Mientras que, el Oxicloruro de Cobre no mostró riesgo a nivel del ambiente acuático, pues el cociente de riesgo (CR) fue menor a 0,5 . 
Tabla 7. Resumen de la evaluación de riesgos ambientales (ERA) de tres plaguicidas sobre Apohyale grandicornis.

\begin{tabular}{cccccccc}
\hline Plaguicida & $\begin{array}{c}\mathrm{CL}_{50} \\
\mathrm{mg} \cdot \mathrm{L}^{-1}\end{array}$ & $\begin{array}{c}\text { Factor de } \\
\text { Seguridad }\end{array}$ & PNEC & $\begin{array}{c}\text { CAE* } \\
\mathrm{mg} \text { i.a.L }\end{array}$ & $\begin{array}{c}\text { Cociente de } \\
\text { Riesgo* } \\
(\mathrm{CR})\end{array}$ & LOC* $^{*}$ & Riesgo \\
\hline Butaclor & 0,019 & 1000 & 0,000019 & 0,10 & 5263,16 & 0,5 & SI \\
$\begin{array}{c}\text { Clorpirifos } \\
\text { Oxicloruro De }\end{array}$ & 0,021 & 1000 & 0,000021 & 0,05 & 2380,95 & 0,5 & SI \\
Cobre & 0,54 & 1000 & 0,00054 & 0,15 & 277,78 & 0,5 & SI \\
\hline
\end{tabular}

${ }^{1}$ Cociente de Riesgo $(\mathrm{CR})=\mathrm{CAE} / \mathrm{CL}_{50} *{ }^{*} \mathrm{CAE}=$ Concentración ambiental estimada. ${ }^{*} \mathrm{LOC}=$ "Level of Concern" (Nivel Crítico).

Tabla 8. Resumen del ERA tomando en cuenta Factor de Riesgo y PNEC de tres plaguicidas sobre Apohyale grandicornis.

\begin{tabular}{cccccc}
\hline Plaguicida & $\begin{array}{c}\mathrm{CL}_{\text {so }} \\
\mathrm{mg} \cdot \mathrm{L}^{-1}\end{array}$ & $\begin{array}{c}\mathrm{CAE}^{*} \\
\mathrm{mg} \mathrm{ia} \cdot \mathrm{L}\end{array}$ & $\begin{array}{c}\text { Cociente De Riesgo }^{\mathrm{I}} \\
(\mathrm{CR})\end{array}$ & LOC $^{*}$ & RIESGO \\
\hline Butaclor & 0,019 & 0,10 & 5,26 & 0,5 & SI \\
Clorpirifos & 0,021 & 0,05 & 2,38 & 0,5 & SI \\
Oxicloruro de Cobre & 0,54 & 0,15 & 0,28 & 0,5 & NO \\
\hline
\end{tabular}

${ }^{1}$ PNEC (Predicted No-Effect Concentration) $=\mathrm{CL}_{50} /$ Factor de seguridad. ${ }^{*} \mathrm{CAE}=$ Concentración ambiental estimada. ${ }^{*}$ Cociente de Riesgo $(\mathrm{CR})=$ CAE/PNEC. ${ }^{*} \mathrm{LOC}=$ "Level of Concern" (Nivel Crítico).

Mientras que en la tabla 8 encontramos que, al tomar en cuenta el factor de seguridad y el PNEC, si se encontró un elevado riesgo a nivel de ambiente acuático para todos los plaguicidas investigados.

\section{DISCUSIÓN}

Butaclor

En la prueba de toxicidad aguda con $A$. grandicornis a las $96 \mathrm{~h}$ se halló una $\mathrm{CL}_{50}$ de $0,019 \mathrm{mg} \cdot \mathrm{L}^{-1}$, un valor que refleja una alta toxicidad al ser comparado con otros autores (Tabla 9) como los de Nwani et al. (2013) que trabajaron con el pez Tilapia zillii Gervais, 1848 en un ensayo de toxicidad a las $96 \mathrm{~h}$ obteniendo un $\mathrm{CL}_{50}$ de 1,25 $(0,60$ a 1,85$) \mathrm{mg} \cdot \mathrm{L}^{-1}$. Tilak et al. (2007) que obtuvieron un $\mathrm{Cl}_{50}$ de 0,25 y $0,55 \mathrm{mg} \cdot \mathrm{L}^{-1}$ (Grado técnico y formulado al 50\%) a las 48 h en la especie de pez Channa punctata Bloch, 1793.

La mayor cantidad de investigaciones que se hacen sobre la toxicidad del Butaclor son utilizando los renacuajos, los que registran valores de $\mathrm{CL}_{50}$ que van de 0,53 a 1,52 $\mathrm{mg} \cdot \mathrm{L}^{-1}$ (Geng et al., 2005, Yin et al., 2008, Liu et al., 2011).
Aunque no se han encontrado registros de investigaciones con anfípodos, si se presentan con otros crustáceos. Paredes \& Anaya (2015) con el camarón de río Cryphiops caementarius Molina, 1782 a las 96 h, presentó rangos de $\mathrm{CL}_{50}$ de 3,18-6,25 mg. $\mathrm{L}^{-1}$. He et al. (2012) obtuvieron un $\mathrm{Cl}_{50}$ de $3,40 \mathrm{mg} \cdot \mathrm{L}^{-1}$ a las $48 \mathrm{~h}$ sobre el cladocero Daphnia carinata King, 1852. Del mismo modo en bases de datos para el Butaclor, se observó un valor de $\mathrm{CL}_{50}$, a las $96 \mathrm{~h}$ de $<0,19 \mathrm{mg} \cdot \mathrm{L}^{-1}$ en pruebas con el crustáceo Americamysis bahía Molenock, 1969 (Lewis et al., 2016). El anfípodo A. grandicornis presentó una mayor sensibilidad a este herbicida que otros crustáceos acuáticos.

Los efectos tóxicos del Butaclor son variados dependiendo del individuo afectado, Geng et al. (2010) muestran que a una concentración de $0,88 \mathrm{mg} \cdot \mathrm{L}^{-1}$, los renacuajos de Rana zhenhaiensis Ye, Fei and Matsui, 1995 presentan aumentos muy significativos en el daño de su ADN por este plaguicida. Esta proporcionalidad entre concentración y genotoxicidad también fue encontrada en el bagre Clarias batrachus Linnaeus, 1758 por Abigail et al. (2015), quienes además citan otros daños causados por el Butaclor en peces tales como: pérdida proteica, efectos adversos en el proceso reproductivo normal e inducción de estrés oxidativo en diversos tejidos debido al descenso del sistema de desintoxicación del glutatión. 
Además, que en el caso de $D$. carinata, este herbicida actúa inhibiendo la síntesis de ácidos grasos de cadena muy larga.

A pesar de la variedad de estudios sobre sus efectos en organismos acuáticos no se encontraron antecedentes sobre sus efectos en anfípodos (Tabla 9). El Butaclor, debido a su ecotoxicidad a concentraciones bajas, a sus aplicaciones frecuentes y al poseer una amplia gama de usos, probablemente represente una amenaza para los organismos que habitan en los cuerpos de agua que reciben la escorrentía con pesticidas.

Tabla 9. Concentración letal media $\left(\mathrm{CL}_{50}\right)$ y Concentración Efectiva media $\left(\mathrm{CE}_{50}\right)$ del Butaclor frente a organismos acuáticos.

\begin{tabular}{|c|c|c|c|c|}
\hline Taxa & Organismo & $\begin{array}{c}\text { Tiempo de } \\
\text { Exposición (h) }\end{array}$ & $\begin{array}{c}\mathrm{CL}_{50} / * \mathrm{CE}_{50} \\
\left(\mathrm{mg} \cdot \mathrm{L}^{-1}\right)\end{array}$ & Referencia \\
\hline Sphaeropleales & Scenedesmus obliquus (Kützing, 1833) & 96 & $*^{*} 2,31$ & He et al. (2012) \\
\hline Perciformes & Channa punctata (Bloch, 1793) & 48 & $* 0,25$ & Tilak et al. (2007) \\
\hline Perciformes & Tilapia zillii (Gervais, 1848) & 96 & 1,25 & Nwani et al. (2013) \\
\hline Anura & Fejervarya limnocharis (Gravenhorst, 1829) & 96 & 0,87 & Liu et al. (2011) \\
\hline Anura & Bufo gargarizans (Cantor, 1842) & 96 & 1.32 & Yin et al. (2008) \\
\hline Anura & Fejervarya limnocharis (Gravenhorst, 1829) & 96 & 1,30 & Geng et al. (2005) \\
\hline Anura & Bufo melanostictus (Schneider, 1799) & 48 & 1,18 & Geng et al. (2005) \\
\hline Anura & Polypedates megacephalus (Hallowell, 1861) & 96 & 1,52 & Geng et al. (2005) \\
\hline Anura & Microbyla ornata (Duméril \& Bibron, 1841) & 96 & 0,53 & Geng et al. (2005) \\
\hline Decapoda & Cryphiops caementarius (Molina, 1782) & 96 & $3,18-6,25$ & $\begin{array}{l}\text { Paredes \& Anaya } \\
\qquad(2015)\end{array}$ \\
\hline Cladocera & Daphnia carinata (King, 1852) & $48 \mathrm{~h}$ & 3,40 & He et al. (2012) \\
\hline
\end{tabular}

\section{Oxicloruro de Cobre}

En la prueba de toxicidad aguda con $A$. grandicornis a las 96 h se halló un $\mathrm{CL}_{50}$ de $0,54 \mathrm{mg} \cdot \mathrm{L}^{-1}$, un valor que refleja una alta toxicidad al ser comparado con los registrados por otros autores en otros individuos (Tabla 10). Ferreira et al. (2004) y Fryday \& Thompson (2012) encontraron para renacuajos de Rana catesbeiana Shaw, 1862 valores similares de $\mathrm{CL}_{50}$ a las $96 \mathrm{~h}\left(2,4\right.$ y $2,83 \mathrm{mg}$ i.a. $\left.\mathrm{L}^{-1}\right)$. Se encuentran también trabajos de toxicidad con juveniles de peces (Cyrino et al. 2004; Gradila, 2015): Con la carpa común Cyprinus angulatus Heckel, $1843\left(\mathrm{CL}_{50}=0,20 \mathrm{mg}\right.$ i.a. $\left.\mathrm{L}^{-1}\right)$ y el pez cebra Danio rerio Hamilton-Buchanan, $1822\left(\mathrm{CL}_{50}=0,152 \mathrm{mg}\right.$ i.a. $\left.\mathrm{L}^{-1}\right)$. Los mismos Cyrino et al. (2004) encontraron valores de $\mathrm{CL}_{50}$ de $1,433 \mathrm{mg}$ i.a. $\mathrm{L}^{-1}$ para el caracol de agua dulce Biomphalaria glabrata Say, 1818 en un ensayo de 48 h.

A pesar de que no se encuentran trabajos sobre la toxicidad del Oxicloruro de Cobre en anfípodos si los hay sobre otros crustáceos. Paredes \& Anaya (2015) trabajaron con el camarón el camarón de río $C$. caementarius y determinaron valores muy alto de $\mathrm{CL}_{50}$ (2 607,72 mg. L$\left.{ }^{1}\right)$. Otros trabajos con Daphnia similis Claus, 1876 y Daphnia magna Straus, 1820 como el de Gradila (2015) en su ensayo agudo de $48 \mathrm{~h}$ halló un $\mathrm{CE}_{50}$ de $0,10 \mathrm{mg}$ i.a. $\mathrm{L}^{-1}$ para D. magna mientras que Cyrino et al. (2004) hizo lo mismo para $D$. similis encontrando un valor similar de $\mathrm{CE}_{50}$ de $0,065 \mathrm{mg}$ i.a. $\mathrm{L}^{-1}$. Mostrándose $A$. grandicornis más tolerante al Oxicloruro de Cobre que otros crustáceos.

Si bien no hay bioensayos específicos con anfípodos utilizando al Oxicloruro de Cobre como tóxico, si existen trabajos sobre toxicidad del Cobre sobre estos. De tal forma, tenemos que para Gammarus pulex Linnaeus, 1758 el $\mathrm{CL}_{50}$ calculado fue de $0,1 \mathrm{mg} \mathrm{Cu} \mathrm{Cu}^{+2} \cdot \mathrm{L}^{-1}$ (Güven et al., 1999), para la especie Echinogammarus olivii $\mathrm{H}$. Milne Edwards, 1830 fue de 0,25 mg Cu.L $\mathrm{L}^{-1}$ (Bat et al., 1999). Tenemos que Ahsanullah \& Florence (1984) calcularon la $\mathrm{CL}_{50}$ para los juveniles y adultos de Allorchestes compressa Dana, 1852 encontrando valores de 0,11 y 0,5 mg Cu. $\mathrm{L}^{-1}$, mostrando los juveniles presentan 4,5 veces más sensibilidad que los adultos. Asimismo, King et al. (2006) realizaron pruebas con Melita awa Barnard, 1972 y Melita matilda Barnard, 1972 obteniendo valores de $\mathrm{CL}_{50}$ de 0,12 y $0,18 \mathrm{mg} \mathrm{Cu} \cdot \mathrm{L}^{-1}$; los mismos autores también hallaron los valores de $\mathrm{CL}_{50}>0,1 \mathrm{mg} \mathrm{Cu} \cdot \mathrm{L}^{-1}$ para Hyale longicornis Haswell, 1879 y Hyale crassicornis Haswell, 1879 que pertenecen a la misma familia que $A$. grandicornis. En el presente estudio se tiene un $\mathrm{CL}_{50}$ a las $96 \mathrm{~h}$ de $0,28 \mathrm{mg} \mathrm{Cu} \cdot \mathrm{L}^{-1}$ siendo este anfípodo ligeramente más resistente al cobre que otros anfípodos.

El oxicloruro de Cobre debe su toxicidad al cobre en su estructura, pues a pesar que es esencial para el 
funcionamiento correcto del organismo un exceso de este puede ser perjudicial. Por ello es necesario entender cómo actúa y cuáles son los efectos del cobre en el anfípodo. Güven et al. (1999) encontraron que la concentración de cobre en el cuerpo del anfípodo $G$. pulex aumenta en proporción a la concentración de cobre en el agua, y comenta que esto puede deberse a una estrategia de acumulación de metales esenciales muy común en crustáceos. King et al. (2006), en sus ensayos llega a la misma relación al relacionar la concentración y acumulación en el cuerpo del anfípodo, y agrega que las especies que acumulan más cobre son las epibentónicas, como lo es A grandicornis; sin embargo, recalcan que entender concretamente la razón de estas relaciones es difícil, puesto que estaría vinculado con las vías de absorción relativas y con la fisiología de las especies de prueba.

Tabla 10. Concentración letal medio $\left(\mathrm{CL}_{50}\right)$ y Concentración Efectiva media $\left(\mathrm{CE}_{50}\right)$ del Oxicloruro de Cobre frente a organismos acuáticos.

\begin{tabular}{ccccc}
\hline Taxa & Organismo & $\begin{array}{c}\text { Tiempo de } \\
\text { Exposición }(\mathrm{h})\end{array}$ & $\begin{array}{c}\mathrm{CL}_{50} /{ }^{*} \mathrm{CE}_{50} \\
\left(\mathrm{mg} \cdot \mathrm{L}^{-1}\right)\end{array}$ & Referencia \\
\hline Cypriniformes & Danio rerio (Hamilton-Buchanan, 1822) & 96 & 0,152 & Cyrino et al. $(2004)$ \\
Cypriniformes & Cyprinus carpio (Linnaeus, 1758) & 96 & 0,20 & Gradila (2015) \\
Pulmonata & Biomphalaria glabrata (Say, 1818) & 48 & 1,433 & Cyrino et al. $(2004)$ \\
Anura & Rana catesbeiana (Shaw, 1862) & 96 & 2,4 & Ferreira et al. (2004) \\
Anura & Rana catesbeiana (Shaw, 1862) & 96 & 2,8 & Fryday \& Thompson (2012) \\
Decapoda & Cryphiops caementarius (Molina, 1782) & 96 & 2607,72 & Paredes \& Anaya (2015) \\
Cladocera & Daphnia magna (Straus, 1820) & 48 & $* 0,29$ & Lewis et al. (2016) \\
Cladocera & Daphnia magna (Straus, 1820) & 48 & $* 0,10$ & Gradila (2015) \\
Cladocera & Daphnia similis (Claus, 1876) & 48 & $* 0,065$ & Cyrino et al. $(2004)$ \\
\hline
\end{tabular}

Kiaune \& Singhasemanon (2011) argumentan que las vías de toxicidad de cobre mejor estudiadas implican la inhibición de las bombas impulsadas por ATP y los canales iónicos, como en el caso de los bioensayos realizados con Artemia, donde el cobre inhibía la actividad de la enzima $\mathrm{Na} / \mathrm{K}$ ATPasa y $\mathrm{Mg}^{2}$ ATPasa. En esta misma línea, Brooks \& Mills (2003) analizaron los efectos del cobre sobre la osmoregulación del anfípodo Gammarus pulex, encontrando que a niveles de cobre de $10 \mathrm{mg} \cdot \mathrm{L}^{-1}$ o más se reduce significativamente la acción de la $\mathrm{Na} / \mathrm{K}$ ATPasa en las branquias, cosa contraria a lo que hallaron con la actividad aglutinante de la $\mathrm{Mg}^{2}$ ATPasa, que se vio menos afectada.

\section{Clorpirifos}

En la prueba de toxicidad aguda con $A$. grandicornis a las $96 \mathrm{~h}$ se halló un $\mathrm{CL}_{50}$ de $0,021 \mathrm{mg} \cdot \mathrm{L}^{-1}$, un valor que refleja una toxicidad baja al ser comparado con los señalados para otros organismos acuáticos (Tabla 11). Diversos estudios se han realizado en peces, anfibios, insectos, etc, para hallar la concentración letal media del Clorpirifos, pero es de nuestro interés los estudios realizados con crustáceos u otros anfípodos.

Trabajos en crustáceos menores como la pulga de agua (Iannacone \& Alvariño, 2000; Palma et al., 2008;
CCME, 2008; FAO, 2002; Giddings et al., 2014) han proporcionado valores de $\mathrm{CL}_{50}$ que van desde 10,48 (Moina macrocopa Straus, 1820) a 0,035 ug. $\mathrm{L}^{-1}$ (Daphnia ambigua Scourfield, 1947), los que han permitido entender la toxicidad que tiene el Clorpirifos en ambientes acuáticos. Otro trabajo sobre crustáceos es el ejecutado por Montagna (2010) que halló un $\mathrm{CL}_{50}$ de $0,046 \mathrm{mg} \cdot \mathrm{L}^{-1}$ para los juveniles del cangrejo Trichodactylus borellianus Nobili, 1896; y en su recopilación de información comentan las $\mathrm{CL}_{50}$ encontradas en otros crustáceos menores como los anfípodos Ampelisca abdita Mills, 1964 (0,00016 mg.L) y Gammarus palustris Bousfield, $1969\left(0,0003 \mathrm{mg} \cdot \mathrm{L}^{-1}\right)$. Indicando que estas diferencias podrían deberse a la dureza del exoesqueleto y al tamaño de las aberturas de las cámaras branquiales, pues influiría en el ingreso de tóxicos al organismo.

Existen más trabajos sobre la toxicidad del Clorpirifos en anfípodos. Como los realizados Leight \& Van dolah (1999) quienes hallaron para el anfípodo estuarino G. palustris rangos de $\mathrm{CL}_{50}$ a las $96 \mathrm{~h}$ de 0,0003 a $0,0053 \mathrm{mg} \cdot \mathrm{L}^{-1}$, y comparando sus resultados con otros estudios realizados en Gammarus lacustris G.O. Sars, $1864\left(0,00011 \mathrm{mg} \cdot \mathrm{L}^{-1}\right)$, Hyalella azteca Saussure, 1858 $\left(0,00015 \mathrm{mg} \cdot \mathrm{L}^{-1}\right)$, Gammarus pseudolimnaeus Bousfield, $1958\left(0,00018 \mathrm{mg} \cdot \mathrm{L}^{-1}\right)$ y Gammarus fasciatus Say, 1818 $\left(0,00032 \mathrm{mg} \cdot \mathrm{L}^{-1}\right)$ encontraron que este anfípodo estuarino 
poseía menor sensibilidad. A su vez, Giddings et al. (2014) para la elaboración de su informe sobre los riesgos para los organismos acuáticos por el uso de Clorpirifos en los Estados Unidos recolectaron información sobre diferentes ensayos en organismos acuáticos, dentro de ellos los anfípodos, sacando una media geométrica para calcular el $\mathrm{CL}_{50}$ en los estudios que usaban la misma especie. De esta manera encontraron rangos de $\mathrm{CL}_{50}$ entre $0,00007 \mathrm{mg} \cdot \mathrm{L}^{-1}$ en $G$. pulex y $0,0029 \mathrm{mg} \cdot \mathrm{L}-$ ${ }^{1}$ en Gammarus fossarum Koch, 1836, valores mucho menores que los encontrados en este estudio para $A$. grandicornis $\left(0,021 \mathrm{mg} \cdot \mathrm{L}^{-1}\right)$. A. grandicornis se mostró unas 210 veces más resistente que $H$. azteca con quien comparte la familia; un resultado similar se encuentra en el estudio elaborado por CCME (2008) con la misma especie, mostrando a $A$. grandicornis unas 250 veces más resistente. Estas diferencias podrían deberse al hábitat en el que habitan estas especies estudiadas, pues son especies de agua dulce mientras que la usada en el presente trabajo es marina, aunque Giddings et al. (2014) advierte que las diferencias en la sensibilidad entre estos grupos son mínimos; por lo que se deberían hacer más estudios sobre esta especie.

El Clorpirifos trabaja mediante su metabolito activo, clorpirifós oxón, el cual inhibe la actividad de la enzima colinesterasa (AChE), esta inhibición provoca la acumulación de acetilcolina en las terminales sinápticas, causando cambios en la transmisión normal delos impulsos nerviosos. En vertebrados e invertebrados acuáticos fueron reportadas distintas expresiones neurológicas tales como irritabilidad, temblores musculares, movimientos bruscos de los apéndices y convulsiones que terminan en insuficiencia respiratoria y la muerte del animal (Montagna, 2010). Sin embargo Giddings et al. (2014) expone que la inhibición de AChE por Clorpirifos es reversible y que en el caso de exposiciones subletales se puede producir una recuperación de esta enzima.

Tabla 11. Concentración letal media $\left(\mathrm{CL}_{50}\right)$ y Concentración Efectiva media $\left(\mathrm{CE}_{50}\right)$ del Clorpirifos frente a organismos acuáticos.

\begin{tabular}{|c|c|c|c|c|}
\hline Taxa & Organismo & Tiempo de Exposición (h) & $\mathrm{CL}_{50} / * \mathrm{CE}_{50}\left(\mathrm{mg} \cdot \mathrm{L}^{-1}\right)$ & Referencia \\
\hline Diptera & Chironomus calligraphus (Goeldi, 1905) & 48 & $0,01556 \pm 0,00406$ & Iannacone \& Alvariño (2000) \\
\hline Salmoniformes & Oncorhynchus clarkii (Richardson, 1836) & 96 & 0,0054 & CCME $(2008)$ \\
\hline Salmoniformes & Oncorhynchus mykiss (Walbaum, 1792) & 96 & 0,0071 & CCME (2008) \\
\hline Perciformes & Stizostedion vitreum (Mitchill, 1818) & 48 & 0,0125 & CCME (2008) \\
\hline Perciformes & Lepomis cyanellus (Rafinesque, 1819) & 36 & 0,0225 & CCME (2008) \\
\hline Mysida & Neomysis integer (Leach, 1814) & 96 & 0,00016 & Giddings et al. (2014) \\
\hline Harpacticoida & Amphiascus tenuiremis (Brady, 1880) & 96 & 0,00147 & Giddings et al. (2014) \\
\hline Decapoda & Palaemonetes pugio (Holthuis, 1949) & 96 & 0,00015 & Giddings et al. (2014) \\
\hline Decapoda & Neocaridina denticulata (De Haan, 1844) & 96 & 0,457 & Giddings et al. (2014) \\
\hline Decapoda & Macrobrachium rosenbergii (De Man, 1879) & 24 & 0,00030 & Giddings et al. (2014) \\
\hline Decapoda & Paratya australiensis (Kemp, 1917) & 96 & 0,00033 & Giddings et al. (2014) \\
\hline Decapoda & Procambarus sp & 96 & 0,00155 & Giddings et al. (2014) \\
\hline Decapoda & Orconectes immunis (Hagen, 1870) & 96 & 0,006 & Giddings et al. (2014) \\
\hline Decapoda & Trichodactylus borellianus (Nobili, 1896) & 96 & 0,04553 & Montagna (2010) \\
\hline Cladocera & Daphnia magna (Straus, 1820) & 48 & 0,00074 & Palma et al. (2008) \\
\hline Cladocera & Daphnia ambigua (Scourfield, 1947) & 48 & 0,000035 & Giddings et al. (2014) \\
\hline Cladocera & Ceriodaphnia dubia (Richard, 1894) & 96 & 0,000054 & Giddings et al. (2014) \\
\hline Cladocera & Moina australiensis (Sars, 1896) & 48 & 0,00010 & Giddings et al. (2014) \\
\hline Cladocera & Daphnia pulex (Linnaeus, 1758) & 72 & 0,00012 & Giddings et al. (2014) \\
\hline Cladocera & Daphnia longispina (O. F. Müller, 1776) & 96 & 0,00030 & Giddings et al. (2014) \\
\hline Cladocera & Daphnia carinata (King, 1852) & 24 & 0,00019 & Giddings et al. (2014) \\
\hline Cladocera & Simocephalus vetulus (Muller, 1776) & 96 & 0,00050 & Giddings et al. (2014) \\
\hline Cladocera & Daphnia magna (Straus, 1820) & 96 & 0,00082 & Giddings et al. (2014) \\
\hline Cladocera & Daphnia magna (Straus, 1820) & 48 & ${ }^{*} 0,00016$ & FAO $(2002)$ \\
\hline Cladocera & Moina macrocopa (Straus, 1820) & 48 & $0,01048 \pm 0,0016$ & Iannacone \& Alvariño (2000) \\
\hline Isopoda & Asellus aquaticus (Linnaeus, 1758) & 96 & 0,00858 & Giddings et al. (2014) \\
\hline Amphipoda & Gammarus pulex (Linnaeus, 1758) & 96 & 0,00007 & Giddings et al. (2014) \\
\hline Amphipoda & Hyalella azteca (Saussure, 1858) & 48 & 0,00010 & Giddings et al. (2014) \\
\hline Amphipoda & Gammarus fossarum (Koch, 1836) & 96 & 0,00290 & Giddings et al. (2014) \\
\hline Amphipoda & Gammarus palustris (Linnaeus, 1758) & 96 & 0,00019 & Giddings et al. (2014) \\
\hline Amphipoda & Gammarus lacustris (G. O. Sars, 1863) & 96 & 0,00011 & Giddings et al. (2014) \\
\hline Amphipoda & Gammarus palustris (Linnaeus, 1758) & 96 & $0,00521-0,0003$ & Leight \& Van Dolah (1998) \\
\hline
\end{tabular}




\section{Dicromato de Potasio}

En la prueba de sensibilidad con Dicromato de Potasio el valor de la $\mathrm{CL}_{50}$ para $A$. grandicornis fue de $13,04 \mathrm{mg} \cdot \mathrm{L}^{-1}$, siendo semejante a lo reportado por otros autores. Abessa \& Sousa (2003) a las $48 \mathrm{~h}$ determinaron un $\mathrm{CL}_{50}$ para el anfípodo Tiburonella viscana J.L. Barnard, 1964 de 11,21 $\pm 3,76 \mathrm{mg} \cdot \mathrm{L}^{-1}$; o los obtenidos por Cesar et al. (2002, 2004), a las $48 \mathrm{~h}$ para el anfípodo Gammarus aequicauda Martynov, 1931: $\mathrm{CE}_{50}=9,5 \pm 2,1 \mathrm{mg} \cdot \mathrm{L}^{-1}$ y CE $\mathrm{CE}_{50}=9,52 \pm$ $4,7 \mathrm{mg} \cdot \mathrm{L}^{-1}$, respectivamente.

A pesar que el bioensayo que realizaron Rudolph et al. (2011) es el que más se ajusta al realizado en esta investigación, es a su vez el que presenta valores más lejanos; los autores trabajaron en un bioensayo de 96 h, con el anfípodo Ampelisca araucana Gallardo, 1963 obteniendo un $\mathrm{CL}_{50}$ de $55,37 \mathrm{mg} \cdot \mathrm{L}^{-1}$.

\section{Riesgo Ambiental}

Torre (2008), expresa que uno de los problemas ambientales en casi todas las áreas agrícolas del mundo, es el de los efectos negativos derivados del uso inadecuado de agroquímicos. Por lo que evaluar los posibles efectos adversos que causan sobre el ambiente es indispensable para ayudar a la regulación de estos. Reforzando esta idea, Iannacone \& Alvarińo (2009), mencionan que la investigación del efecto ecotoxicológico proporciona más solidez a la evaluación de la calidad de agua, ayudando a que se establezcan mejores criterios de calidad para la protección de la vida acuática.

Así, la evaluación de riesgos se perfila según Torre (2008) como una herramienta que puede utilizarse para estimar y jerarquizar la importancia ambiental de una medida, calculando cuantitativamente y/o cualitativamente los daños a los ecosistemas derivados de la exposición a un contaminante ambiental. Por su parte Vassiliou (2016) en su estudio advierte la importancia de, además de los factores de exposición, toxicidad y las características del ecosistema, incluir los que afectan la fuente, el patrón de aplicación, la carga y la disponibilidad de plaguicidas, para la evaluación del riesgo de los plaguicidas en cuerpos de agua.

La evaluación del riego realizado en esta investigación entra en lo que Peterson (2006) categoriza como los de Nivel 1; que si bien se caracterizan por suposiciones de toxicidad y exposición muy conservadoras, y que se utilizan principalmente para descartar riesgos insignificantes en la toma de decisiones regulatorias, son valiosos para hacer comparaciones directas del riesgo cuantitativo entre plaguicidas.
Referente a esto Vassiliou (2016) comenta que es tan complejo y variados los escenarios y factores que afectan el riesgo, que las nuevas tendencias en las pruebas de toxicidad tienden a modificar el enfoque clásico de un procedimiento de evaluación de riesgos.

Dada la importancia mencionada en la utilización de uso del cociente de riesgo y otros factores dentro de la evaluación del riesgo, para determinar la peligrosidad de una sustancia, es contradictorio que en el Perú no se tome en cuenta esto, para la regulación de plaguicidas; pues en el decreto supremo (DS, 2015) extendido por el MINAGRI (Ministerio de Agricultura y Riego), donde se habla sobre los requisitos generales para la evaluación o revaluación de plaguicidas químicos de uso agrícola. Se mencionan las especificaciones para los ensayos agudos y crónicos; sin embargo, para la evaluación del riesgo en plaguicidas no hay especificaciones. Lo que podría ser fruto del poco manejo, conocimiento e importancia de este tipo de pruebas en la regulación peruana de plaguicidas.

Se concluye que: (1) existe sensibilidad del anfípodo $A$. grandicornis frente a los plaguicidas estudiados (Butaclor, Clorpirifos y Oxicloruro de Cobre) en ensayos agudos. Perfilándose así como una buena opción de bioindicador para ensayos de este tipo. (2) según su toxicidad, a las $96 \mathrm{~h}$, los plaguicidas evaluados pueden ser ordenados de manera descendente de la siguiente manera: Butaclor $\left(0,019 \mathrm{mg} \cdot \mathrm{L}^{-1}\right)>$ Clorpirifos $\left(0,021 \mathrm{mg} \cdot \mathrm{L}^{-1}\right)>$ Oxicloruro de Cobre $\left(0,54 \mathrm{mg} \cdot \mathrm{L}^{-1}\right)$. Siendo el Butaclor 1,11 veces más tóxico que el Clorpirifos y unas 28,42 veces más tóxico que el Oxicloruro de Cobre. (3) el Dicromato de Potasio afecta a la mortandad de $A$. grandicornis a las 24, 48, 72 y $96 \mathrm{~h}$, presentando a las $96 \mathrm{~h}$ un $\mathrm{CL}_{50}$ de $13,04 \mathrm{mg} \cdot \mathrm{L}^{-1}$. De lo que se concluye que este tóxico de referencia puede utilizarse para test de sensibilidad en $A$. grandicornis.

Por otro lado, se tienen aún tareas por ejecutar, por lo que se debe efectuar más bioensayos de toxicidad aguda y crónica con Butaclor sobre anfípodos, debido a la escasa bibliografía referida a esta especie. Es conveniente realizar trabajos sobre la toxicidad del cobre en $A$. grandicornis utilizando otros compuestos cúpricos. También sería bueno desarrollar más pruebas con $A$. grandicornis usando otras sustancias químicas. Igualmente, se debe comprobar si la zona de muestreo de $A$. grandicornis afecta, de alguna manera en su sensibilidad a ciertos tóxicos. Es necesario complementar estos estudios de toxicidad aguda del Butaclor con trabajos sobre su efecto en la fisiología y comportamiento de A. grandicornis. Hay que realizar bioensayos de sensibilidad con $A$. grandicornis usando otras sustancias de referencias, para poder comparar a mayor escala su sensibilidad. Finalmente, se deben 
realizar estudios más profundos sobre el riesgo ambiental que implica el uso de estos plaguicidas en el ambiente acuático.

\section{REFERENCIAS BIBLIOGRÁFICAS}

Abessa, D. \& Souza, E. 2003. Sensitivity of the Amphipod Tiburonella viscana (Platyischnopidae) to $\mathrm{K}_{2} \mathrm{CR}_{2} \mathrm{O}_{7}$. Brazilian Archives of Biology and Technology, 46: 53-55.

Abigail, M.; Samuel, S. \& Ramalingam, C. 2015. Addressing the environmental impacts of butachlor and the available remediation strategies: a systematic review. International Journal of Environmental Science and Technology, 12: 4025-4036.

Ahsanullah, M. \& Florence, T. 1984.Toxicity of copper to the marine amphipod Allorchestes compressa in the presence of water- and lipid-soluble ligands. Marine Biology, 84: 41-45.

Albert, L.A. 2005. Panorama de los plaguicidas en México. Revista de toxicología en línea. Recuperado de: http://www.sertox.com.ar/retel/default.htm.

Bat, L.; Gündogdu, A.; Sezgin, M.; Çulha, M.; Gönlügür, G. \& Akbulut, M. 1999. Acute toxicity of zinc, copper and lead to three species of marine organisms from the Sinop Peninsula, Black Sea. Tr. Journal of Biology, 23: 537-544.

Bousfield, E.L. \& Hendrycks, E.A. 2002. The Talitroidean Amphipod family Hyalidae revised, with emphasis on the North Pacific fauna: systematics and distributional ecology. Amphipacifica, 3: 17-134.

Brooks, S.J. \& Mills, C.L. 2003. The effect of copper on osmoregulation in the freshwater amphipod Gammarus pulex. Comparative Biochemistry and Physiology, 135: 527-537.

Cadien, D. 2015 Amphipoda of the Northeast Pacific (Equator to Aleutians, intertidal to abyss): II. Talitroidea - a review. Southern California. Association of Marine Intertidal Taxonomists. $45 \mathrm{pp}$.

CCME (Canadian council of ministers of the environment). 2008. Canadian water quality guidelines for the protection of aquatic life: Chlorpyrifos. Canadian Environmental Quality Guidelines.

Cesar, A.; Marín, A.; Marín-Guirao, L. \& Vita, R. 2004. Amphipod and sea urchin tests to assess the toxicity of Mediterranean sediments: the case of Portmán Bay. Scientia Marina, 68 (Suppl. 1): 205-213

CA (Comunidad Andina). 2002. Manual técnico andino para el registro y control de plaguicidas quimicos de uso agrícola. Gaceta Oficial. 169pp.

Cyrino, E.; Matos, R. \& Roma. F. 2004. Comparative study on the susceptibility of freshwater species to copper-based pesticides. Chemosphere, 56: 369-374.

Chapman, J.W. 2007. Gammaridea. En: Carlton, J.T. (Ed). The Light and Smith Manual: Intertidal Invertebrates from Central California to Oregon. $4^{\text {th }}$ ed. University of California Press, Berkeley, California. pp. 545-611.

DS (Decreto supremo). 2015. DS. No 001. MINAGRI. Diario "El Peruano": Normas Legales. 29 de Enero del 2015.

Fairchild, W.L. ; Doe, K.G.; Jackman, P.M.; Arsenault, J.T. ; Aubé, J.G. ; Losier, M. \& Cook, A.M. 2010. Acute and chronic toxicity of two formulations of the pyrethroid pesticide deltamethrin to an amphipod, sand shrimp and lobster larvae. Canadian Technical Report of Fisheries and Aquatic Sciences, 2876: 1-34.

FAO (Food and Agriculture Organization of the United Nations). 2002. FAO specifications and evaluations for agricultural pesticides: Chlorpyrifos. FAO.

Ferreira, C.; Lombardi, J.; Machado, J.; Bueno, H.; Soares, S. \& Saldiva, P. 2004. Effects of copper oxychloride in Rana Catesbeiana tadpoles: toxicological and bioaccumulative aspects. Bulletin of Environmental Contamination and Toxicology, 73: 465-470.

Finney, D.J. 1952. Probit Analysis (2nd Ed). Journal of the Institute of Actuaries, 78: 388-390.

Fryday, S. \& Thompson, H. 2012. Toxicity of pesticides to aquatic and terrestrial life stages of amphibians and 
occurrence, habitat use and exposure of amphibian species in agricultural environments. EFSA Publications, External Scientific Report 343, 9:9.

Geng, B.; Yao, D. \& Xue, Q. 2005. Acute toxicity of the pesticide dichlorvos and the herbicide butachlor to tadpoles of four anuran species. Bulletin of environmental contamination and toxicology, 75: 343-349.

Geng, B.; Lin, L.; Zhang, Q. \& Zhong, B. 2010. Genotoxicity of the pesticide Dichlorvos and herbicide Butachlor on Rana zhenhaiensis tadpoles. Asian Herpetological Research, 1: 118-122.

Giddings, J.M.; Williams, W.M.; Solomon, K.R. \& Giesy, J.P. 2014. Risks to aquatic organisms from use of Chlorpyrifos in the United States. Reviews of Environmental Contamination and Toxicology, 231: 119-162.

González, E.R. 1991. The genus Hyale in Chile. Spixiana, 14:125-142.

Gradila, M. 2015. Environmental risk assessment of some copper based fungicides according to the requirements of good laboratory practice. AgroLife Scientific Journal, 4: 74-78.

Guerrero, J. 2007. Estudio comparativo de tratamientos herbicidas post - emergentes sobre el complejo de malezas en arroz de riego (Tesis de grado). Universidad técnica de Babahoyo. Babahoyo. Ecuador.

Güven, K.; Özbay, C.; Ünlü, E. \& Satar, A. 1999. Acute lethal toxicity and accumulation of copper in Gammarus pulex (L.) (Amphipoda). Turkish Journal of Biology, 23: 513-522.

He, H.; Yu, J.; Chen, G.; Li, W.; He, J. \& Li, H. 2012. Acute toxicity of butachlor and atrazine to freshwater green alga Scenedesmus obliquus and cladoceran Daphnia carinata. Ecotoxicology and Environmental Safety, 80: 91-96.

Iannacone, J. \& Alvariño, L. 2000. Chironomus calligraphus Goeldi y Moina macrocopa Sars como herramientas ecotoxicológicas para la evaluación de Lindano y Clorpirifos. Boletín de la Sociedad de Biología de Concepción, 71: 73-79.
Iannacone, J.; Caballero, R. \& Alvariño, I. 2002. Empleo del caracol de agua dulce Physa venustula Gould como herramienta ecotoxicológica para la evaluación de riesgos ambientales por plaguicidas. Agricultura Técnica (Chile), 62: 212-225.

Iannacone, J. \& Alvariño, L. 2002. Evaluación del riesgo ambiental del insecticida Cartap en bioensayos con tres invertebrados. Agricultura Técnica (Chile), 62: 366-374.

Iannacone, J. \& Alvariño, L. 2004. Eisenia foetida (Savigny, 1826) (Annelida: Lumbricidae) como modelo ecotoxicológico para evaluar lindano y clorpirifos. Acta Zoológica Lilloana, 48: 5-12.

Iannacone, J. \& Alvariño, L. 2009. Evaluación del riesgo acuático de siete productos farmacéuticos sobre Daphnia magna. Ecología Aplicada, 8: 71-80.

King, C.K.; Gale, S.A.; Hyne, R.; Stauber, L.J.V.; Simpson, S.L. \& Hickey, S.W. 2006. Sensitivities of Australian and New Zealand amphipods to copper and zinc in waters and metal-spiked sediments. Chemosphere, 63: 1466-1476.

Kiaune, L. \& Singhasemanon, N. 2011. Pesticidal Copper (I) Oxide: Environmental Fate and Aquatic Toxicity. In: Whitacre, D.M. (ed.), Reviews of Environmental Contamination and Toxicology, Volume 213. pp. 1-26.

Lasheidani, M.; Balouchi, S.; Keyvan, A.; Jamili, S. \& Falakrou, K. 2008. Efectos de Butachlor la densidad, el volumen y el número de espermatozoides anormales en Caspian Kutum ( Rutilus frisii kutum, Kamenskii 1901). Investigación Revista de Ciencias del Medio Ambiente, 2: 474-482.

Leight, A. \& Van Dolah, R. 1999. Acute toxicity of the insecticides Endosulfan, Chlorpyrifos, and Malathion to the epibenthic estuarine amphipod Gammarus palustris (Bousfield). Environmental Toxicology and Chemistry, 18: 958-964.

Lewis, K.; Tzilivakis, J.; Warner, D. \& Green, A. 2016. An international database for pesticide risk assessments and management. Human and Ecological Risk Assessment: An International Journal, 22: 1050-1064. 
Liu, W.; Wang, C.; Wang, T.; Fellers, G. ; Lai, B. \& Kam, Y. 2011. Impacts of the herbicide Butachlor on the larvae of a paddy field breeding frog (Fejervarya limnocharis) in subtropical Taiwan. Ecotoxicology, 20: 377-384.

Montagna, M.C. 2010. Toxicidad de clorpirifos como elemento activo de un formulado comercial en juveniles del cangrejo Trichodactylus borellianus. Natura Neotropicalis, 1: 31-43.

Navarro, C. 2015. Anfipodos en cuevas submarinas del sur de la Península Ibérica (Tesis Doctoral). Universidad de Sevilla. Sevilla, España.

Nwani, C.; Ama, U.; Okoh, F.; Oji, U.; Ogbonyealu, R.; Ibiam, A. \& Udu-Ibiam, O. 2013. Acute toxicity of the chloroacetanilide herbicide butachlor and its effects on the behavior of the freshwater fish Tilapia zillii. African Journal of Biotechnology, 12: 499-503.

Paredes, A. \& Anaya, R. 2015. Efecto agudo del Oxicloruro de Cobre y del Butaclor sobre el "Camarón de Río" Cryphiops caementarius (Molina 1782). Ecología Aplicada, 14: 71-74.

Palma, P.; Palma, V.; Fernandes, R.; Soares, A. \& Barbosa I. 2008. Acute toxicity of Atrazine, Endosulfan Sulphate and Chlorpyrifos to Vibrio fischeri, Thamnocephalus platyurus and Daphnia magna, relative to their concentrations in surface waters from the Alentejo Region of Portugal. Bulletin of Environmental Contamination and Toxicology, 81:485-489.

Peluso, M.L. 2011. Evaluación de efectos biológicos y biodisponibilidad de contaminantes en sedimentos del Río de la Platay afluentes (Tesis doctoral). Universidad Nacional de la Plata, La Plata, Argentina.

Pérez, J. 2009. Composición y diversidad de la fauna de Amphipoda (Crustacea, Aeracarida) asociada a instalaciones acuicolas de Bahia Metri, Región de los Lagos, Chile. Centro de Estudios en Biodiversidad (CEBCh). Osorno, Chile.

Pérez, W. \& Forbes, G. 2007. ¿Qué es un Fungicida? División de manejo integrado de cultivos. Hoja divulgativa, 5:4.

Peterson, R.K. 2006. Comparing ecological risks of pesticides: the utility of a Risk Quotient ranking approach across refinements of exposure. Pest Management Science, 62: 46-56.

Planes, E. \& Fuchs, J. 2015. Cuáles son los aportes de la ecotoxicología a las regulaciones ambientales. Ciencia e Investigación, 65: 45-62.

Rudolph, A.; Medina, P.; Ahumada, R. \& Novoa, V. 2011. Calidad ecotoxicológica de los sedimentos en fiordos del sur de Chile. Revista de biología marina y oceanografía, 46: 79-84.

Serejo, C.S. 2003. Cladistic revision of talitroidean amphipods (Crustacea, Gammaridea), with a proposal of a new classification. Zoologica Scripta, 33: 551-586.

Tilak, K.; Veeraiah, K.; Bhaskara-Thathaji, P. \& Butchiram, M. 2007. Toxicity studies of butachlor to the freshwater fish Channa punctata (Bloch). Journal of Environmental Biology, 28: 485-487.

Torre, D.A. 2008. Propuesta metodológica para el análisis de riesgo ambiental en sistemas productivos agricolas bajo invernadero en Colombia (Tesis de Grado). Universidad de la Salle. Bogotá, D.C. Colombia.

USEPA (United States Environmental Protection Agency). 1994. Methods for assessing the toxicity of sediment-associated contaminants with estuarine and marine Amphipods. Washington: Environmental Agency Protecion.

USEPA (United States Environmental Protection Agency). 2002. Guidelines for Ecological Risk Assessment. Washington: Environmental Agency Protection.

Vassiliou, G. 2016. Factors affecting risk assessment of pesticides in water bodies: A review. MOJ Toxicology, 2: 26-32.

Yin, X.; Li, S.; Zhang, L.; Zhu, G. \& Zhuang, H. 2008. Evaluation of DNA damage in Chinese toad (Bufo gargarizans) after in vivo exposure to sublethal concentrations of four herbicides using the comet assay. Ecotoxicology, 17: 280286.

Received August 22, 2019.

Accepted October 30, 2019. 